\title{
Call for Papers
}

\section{European Psychologist Special Section on Ambulatory Assessment}

The primary intention of the forthcoming special section is to provide the audience a concise collection of state of the art reviews on ambulatory assessment. In particular, we want to bring together different approaches and traditions of ambulatory assessment from various disciplines within psychology. The following issues should be included in the manuscripts: (a) A brief overview of line of research and traditions. (b) Selected fields of research in each discipline should be covered, where ambulatory assessment methods have proved to provide insights to research questions that could not be adequately answered by lab-based or cross-sectional/longitudinal survey research only. Benefits and strengths, but also pitfalls and drawbacks, of ambulatory monitoring methodology should be highlighted and critically appreciated. (c) Future prospects should be outlined.

Submissions for consideration in this special section should be sent to any one of the action editors: Ulrich Ebner-Priemer, Central Institute of Mental Health, Mannheim, Germany (E-mail ulrich.ebner-priemer@zi-mannheim.de), Kurt Pawlik, University of Hamburg, Germany (E-mail pawlik@uni-hamburg.de), or Thomas Kubiak, University of Greifswald, Germany (E-mail kubiak@unigreifswald.de). Manuscript should not exceed 7500 words of main text plus ca. 40 references.

Submission deadline is January 31, 2008. For more information, please contact any of the action editors as given above. 